\title{
Realistic Numerical and Analytical Modeling of Light Scattering in Brain Tissue for Optogenetic Applications ${ }^{1,2,3}$
}

\author{
Guy Yona, ${ }^{1}$ Nizan Meitav, ${ }^{2}$ - Itamar Kahn, ${ }^{3}$ and Shy Shoham ${ }^{2}$
}

DOI:http://dx.doi.org/10.1523/ENEURO.0059-15.2015

${ }^{1}$ Autonomous Systems Program (TASP), ${ }^{2}$ Faculty of Biomedical Engineering, and ${ }^{3}$ Rappaport Faculty of Medicine and Institute, Technion - Israel Institute of Technology, Haifa, 31096, Israel

\begin{abstract}
In recent years, optogenetics has become a central tool in neuroscience research. Estimating the transmission of visible light through brain tissue is of crucial importance for controlling the activation levels of neurons in different depths, designing optical systems, and avoiding lesions from excessive power density. The Kubelka-Munk model and Monte Carlo simulations have previously been used to model light propagation through rodents' brain tissue, however, these prior attempts suffer from fundamental shortcomings. Here, we introduce and study two modified approaches for modeling the distributions of light emanating from a multimode fiber and scattering through tissue, using both realistic numerical Monte Carlo simulations and an analytical approach based on the beam-spread function approach. We demonstrate a good agreement of the new methods' predictions both with recently published data, and with new measurements in mouse brain cortical slices, where our results yield a new cortical scattering length estimate of $\sim 47 \mu \mathrm{m}$ at $\lambda=473 \mathrm{~nm}$, significantly shorter than ordinarily assumed in optogenetic applications.
\end{abstract}

Key words: beam-spread function; light scattering; Monte Carlo optogenetics

\section{Significance Statement}

For optogenetic stimulation to become highly controlled, reproducible, and safe, a thorough understanding of the deep-tissue scattered-light distributions that mediate the excitation is required. However, effective computation tools validated by actual measurements in brain tissue are currently still lacking. In this paper, we introduce, study, and validate new numerical and analytical approaches for modeling the distributions of light propagating through brain tissue. We show that both methods lead to consistent results and use the much faster analytical method to iteratively extract the optical parameters from new measurements, suggesting that light penetration into cortical tissue is significantly less than usually assumed. The new level of faithfulness could assist in designing experimental setups and optical interfaces, and help interpret optogenetic experiments.

\section{Introduction}

Optogenetic neuromodulation is playing an increasingly central role in neuroscience research and emerging ap-

Received May 31, 2015; accepted October 30, 2015; First published January 4, 2016.

${ }^{1}$ The authors report no conflict of interest.

${ }^{2}$ Contributions: G.Y., I.K., and S.S. designed research; G.Y. and N.M. performed research; G.Y. and S.S. analyzed data and wrote the paper. plications (Deisseroth, 2011), with major efforts being directed toward the discovery and development of advanced optogenetic probes (Yizhar et al., 2011; Zhang et al., 2011) and related miniature devices (Deisseroth and Schnitzer, 2013). However, relatively little attention has been

\footnotetext{
${ }^{3}$ This work was supported by a grant from the Israel Ministry of Science, Technology and Space, ISF 225/11 (I.K.) and FP7-IRG PCIG9-GA-2011294001 (I.K.), and G.Y. is supported in part by the Gutwirth Family fund.
} 
given to elucidating and characterizing the passage of light in brain tissue at the relevant visible wavelengths and illumination geometries: because light-tissue interactions at these wavelengths is strongly scattering-dominated, light transfer is heavily affected by multiple scattering events resulting in complex light distributions where the photons deviate considerably from their original directions. Estimating the resulting light distribution is of crucial importance for multiple aspects of optogenetic research including the control and analysis of neuronal excitation levels, comparison of the relative merits for different applications of probes with different excitation spectra, the design of effective optical systems for delivering sufficient light power density to target regions (Pashaie et al., 2014), and for avoiding lesions and light toxicity (Frigault et al., 2009) that may result from excessive light absorption.

Despite this central importance in interpreting and designing optogenetic experiments, methodical treatment of tissue light transport in this context has been sparse. To date, the central approaches used for studying relevant scattered light distributions in rodent brains were based on a Kubelka-Munk (KM) model fit to empirical results (Aravanis et al., 2007; Adamantidis et al., 2007; Yizhar et al., 2011; Foutz et al., 2012), and on Monte Carlo (MC) simulations of light transport (Bernstein et al., 2008; Chow et al., 2010; Kahn et al., 2011). Unfortunately, the generality and applicability of each of these approaches suffers from major limitations. The KM model (Kubelka, 1948) is a one-dimensional model describing the propagation of light through a diffuse scattering medium (with no absorption), based on two coupled differential equations that describe the change in the intensity at each point in the slab based on the change of two fluxes: the transmitted light flux and the backscattered flux. However, the KM model is based on fundamental assumptions that are inconsistent with light scattering in tissue geometries and length scales relevant to optogenetics: (1) it assumes isotropic scattering, which becomes true only at the diffusive regime, depths of multiple millimeters, whereas at the distance scales and wavelengths relevant to optogenetics, scattering is highly anisotropic; and (2) it assumes isotropic illumination (i.e., illumination from an infinite uniform plane), thus neglecting the finite geometry and size of the illumination, which is typically of comparable size to the tissue of interest. The various limitations of the KM model, as suitable for capturing light scattering, are extensively discussed by Neuman and Edström (2010). Likewise, the published MC calculations (Bernstein et al.,

We thank Prof. Ofer Yizhar for helpful discussions, Dr Inbar Brosh for her technical assistance in producing the brain slices for the experiment, Shir Paluch for assistance with the BSF implementation, and Eliran Guzi and Dalia Rifold for writing the simulation GUI.

Correspondence should be addressed to Shy Shoham, Department of Biomedical Engineering, Technion - Israel Institute of Technology, Haifa 32000, Israel. E-mail: shy@technion.ac.il

DOI:http://dx.doi.org/10.1523/ENEURO.0059-15.2015

Copyright (C) 2016 Yona et al.

This is an open-access article distributed under the terms of the Creative Commons Attribution 4.0 International, which permits unrestricted use, distribution and reproduction in any medium provided that the original work is properly attributed.
2008; Chow et al., 2010; Kahn et al., 2011) were based on scattering parameters for human brains (Yaroslavsky et al., 2002), and are unvalidated by empirical data (see Discussion). More recently, more extensive sets of measurements for estimating the optical parameters using different experimental strategies were performed in several subcortical adult mouse brain areas (Al-Juboori et al., 2013) and in adult rat brains (Azimipour et al., 2014). Although important, broad empirical measurement datasets do not provide the type of generality required for tackling a diversity of practical cases and designs, and leaves open the need for a complementary quantitative, practical, and empirically validated modeling framework for optogenetic light propagation.

In this work, we apply two independent methods for detailed modeling of the transmission of light launched from a monochromatic optical fiber light source across various thicknesses of brain tissue: the MC method (Fang, 2010) and the beam spread function (BSF) method (McLean et al., 1998), which is applied in this context for the first time. MC simulation uses repeated numerical random sampling, whereas BSF is based on convolutions of analytical beam propagation Green's function (an impulse response, which is used via the superposition principle to obtain a solution to more complex initial or boundary conditions). Despite the very different methodologies behind these two methods, we show that their results are both mutually consistent and in agreement with both published and new empirical brain-scattering results. Finally, we discuss the new approaches' relative merits and limitations, as well as directions for future study.

\section{Methods}

\section{General considerations}

In our models and in previous experiments (Aravanis et al., 2007; Yizhar et al., 2011), an optical fiber was located adjacent and perpendicular to an ex-vivo slice of mouse brain tissue. The fiber was emitting light in wavelength $\lambda=473 \mathrm{~nm}$, its core radius was $100 \mu \mathrm{m}$ and numerical aperture (NA) of 0.37 . These parameters were also used by Aravanis et al. (2007). We assume that the spatial and angular intensity distributions of the emitted light at the fiber surface are uniform and constant. This assumption holds because the electric field distribution at the fiber tip is composed of superposition of all the linearly polarized modes (amplitude distributions that remain unchanged during propagation in a fiber) supported by the fiber, which are very numerous. The number of modes $(M)$ is typically estimated from the fiber's normalized frequency $V=2 \pi\left(r_{\text {core }} / \lambda\right) N A=491.5$; (Okamoto, 2006), which indeed implies a very large number of modes $M \approx 4 V^{2} / \pi^{2} \approx 10^{5}$.

Another important parameter is the beam divergence at the fiber tip, because it determines the angular distribution of the light emanating from each point at the fiber tip. If we assume that the fiber is touching the tissue (i.e., no other interface between them), the half-angle of divergence $\theta_{\text {div }}$ is 


$$
\theta_{\text {div }}=\sin ^{-1}\left(\frac{N A}{n_{\text {tissue }}}\right)=15.8^{\circ},
$$

where $n_{\text {tissue }}=1.36$ is the refractive index of the brain tissue (Vo-Dinh, 2003). We assume a homogenous and isotropic (i.e., there is no preferable direction in the tissue) scattering tissue, characterized by a scattering coefficient $\mu_{s}\left(\mathrm{~cm}^{-1}\right)$, a weak absorption coefficient $\mu_{a}\left(\mathrm{~cm}^{-1}, \mu_{a} \ll\right.$ $\left.\mu_{s}\right)$, and a high anisotropy coefficient $g$ (dimensionless) which represents a tendency for strongly forward scattering at each scattering event. Using the two methods described below, we calculate local intensity (the radiant power passing through a unit surface) in different points across the tissue.

\section{Monte Carlo simulation}

The MC simulation is based on the Mesh-based Monte Carlo (MMC) code developed by Fang (2010), version 0.9.5. Because the MMC software is based on an infinitely narrow beam (pencil beam) light source, we adapted it to a more complex light source using a three-step process:

(a) The 3D pencil-beam response was produced by simulations performed at a logarithmic lateral resolution spanning from $1.2 \mu \mathrm{m}$ near the central beam to $47 \mu \mathrm{m}$ at the edge of the simulated volume, and an axial resolution of $5 \mu \mathrm{m}$, with $10^{8}$ simulated photons. The response was then resampled by a uniform isotropic grid of $5 \mu \mathrm{m}$.

(b) The 3D pencil-beam response was rotated in 64 intervals over the inclination angle $\theta_{\text {div }}$ along the rotation axis, which is the entrance point of the pencil beam into the tissue. Sequentially, the result was rotated over a full circle in 64 intervals along the azimuthal direction. The results of all the rotations were summed up to form the angular light pattern that was emitted from each point of the fiber tip (Fig. 2a). In effect, the rotations can be formulated in terms of angular convolution with unit vectors that span the inclination angles $\left[0, \theta_{\text {div }}\right]$ and the azimuth angles $[0,2 \pi]$.

(c) To take into account the fiber-tip area, the result of the previous stage was convolved with a sampled disk of the same diameter as the fiber (Fig. 2a).

\section{Beam-spread function simulation}

The BSF method (McLean et al., 1998) is a uniquely powerful solution for approximating light distributions in highly forward scattering media where the higher-order effects of photons coming via multiple paths of various lengths, thus resulting in time dispersion of the light intensity, is also incorporated. The method applies an analytical approximation for unidirectional pulsed source propagation in a turbid medium, which serves as a Green's function that can be used to solve more general problems (i.e., via angular and spatial convolution): the BSF $k(z, \boldsymbol{\rho}, \tau)$ is the intensity distribution of light from a pulsed source normalized to the pulse's energy, after propagating a distance $z$ in the medium, where $\boldsymbol{\rho}$ is the radial position vector, and $\tau=t-z / c$ is the multipath time ( $c$ is the speed of light in the medium, $t$ is the time since the photons start propagating, $\tau=0$ for unscattered photons). McLean et al. (1998) present a useful decomposition of the scattered photons BSF into a product of a normalized temporal dispersion distribution function $G(z, \tau)$ and a normalized spatial-angular distribution function $h(z, \boldsymbol{\rho}, \tau)$ (Fig. 1, top):

$$
k_{s c}(z, \boldsymbol{\rho}, \tau)=e^{-\mu_{a}(z+c \tau)} G(z, \tau) \cdot h(z, \boldsymbol{\rho}, \tau),
$$

where $\mu_{a}$ is the absorption coefficient. Following McLean et al. (1998), we use the time-dependent spatial distribution function (derived from the time-independent smallangle approximation):

$$
h(z, \boldsymbol{\rho}, \tau)=\frac{3}{4 \pi \tau c z} \exp \left(-\frac{3}{4} \frac{\boldsymbol{\rho}^{2}}{\tau c z}\right),
$$

and the Gamma probability density function is used as the temporal distribution function (equivalent to a normal distribution when the variable, $\tau$, is positive definite):

$$
G(z, \tau)=\frac{\mu}{\sigma^{2} \Gamma\left(\frac{\mu^{2}}{\sigma^{2}}\right)}\left(\frac{\mu \tau}{\sigma^{2}}\right)^{\frac{\mu^{2}}{\sigma^{2}}} \exp \left(-\frac{\mu \tau}{\sigma^{2}}\right),
$$

where $\mu$ and $\sigma$ are the first and second moments of $\tau$, respectively, and are dependent only on the first and second moments of the cosine of the scattering angle $\theta$ (McLean et al., 1998 provides the formulas), and $\Gamma(x)$ is the gamma function. The BSF of the scattered photons, $k_{s c}(z, \rho, \tau)$, was calculated for the tissue optical parameters (same as for the MC method) and integrated over time. The unscattered photons were added next to obtain the combined BSF (Fig. 1, bottom):

$$
k(z, \boldsymbol{\rho}, \tau)=\delta(\boldsymbol{\rho}) \delta(\tau) e^{-\left(\mu_{a}+\mu_{s}\right) z}+\left(1-e^{-\mu_{s} z}\right) k_{s c}(z, \boldsymbol{\rho}, \tau) .
$$

Subsequently, angular and spatial convolutions were performed to obtain the propagation of light from the fiber's tip, as was done with the MC method (only Steps 2 and 3 are required here, because the Green's function is calculated for the entire volume; Fig. 2).

The calculation of the BSF pencil beam is considerably faster than MC calculation with comparable accuracy (several seconds compared to hours). As a result, curve fitting can be more readily used to obtain the optical parameters of a sample. We implemented the accelerated gradient search method (Beck and Teboulle, 2009) to find the scattering coefficient and anisotropy factor of the published data manually extracted from Aravanis et al. (2007) and of our measurements (see Fig. 5).

\section{Experimental setup}

All procedures were conducted in accordance with the national ethics committee for animal experimentation and with the approval of the Committee on Animal Care at The Technion-Israel Institute of Technology. Mouse brain slices were illuminated from above with a blue laser ( 473 $\mathrm{nm}$ ) emanating from a fiber of $200 \mu \mathrm{m}$ in diameter with NA of 0.37 (Thorlabs BFL37-200). The light intensity was measured through a small aperture $(\sim 50 \mu \mathrm{m}$ diameter) using a power meter (Newport 818-ST), when the fiber tip 


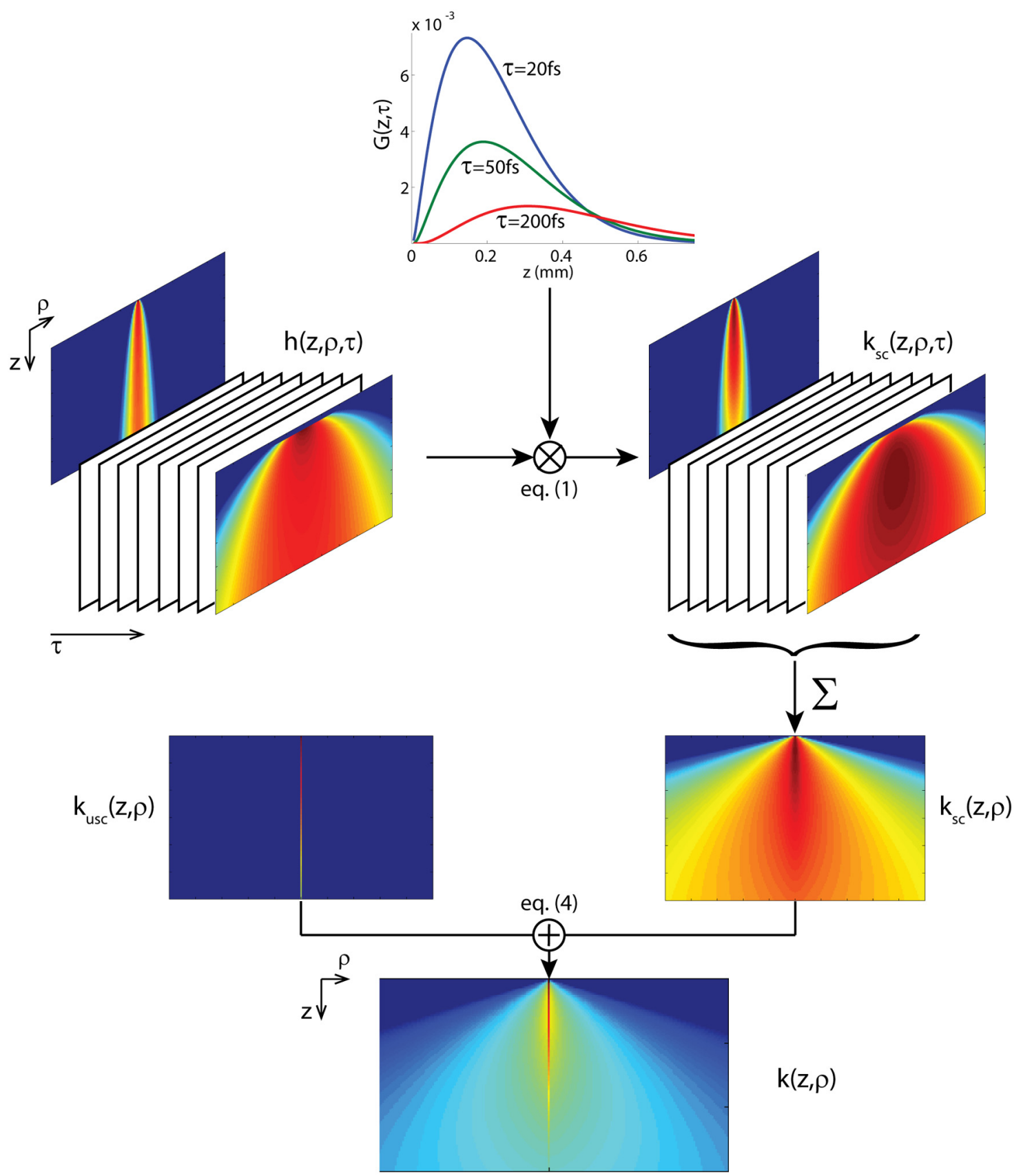

Figure 1. Graphical depiction of the BSF calculation process: the distribution of the scattered photons $k_{\mathrm{sc}}(z, \boldsymbol{\rho}, \tau)$ is a product of the spatial distribution $h(z, \boldsymbol{\rho}, \tau)$ and the temporal dispersion distribution $G(z, \tau)$ (Eq. 1). The time-dependent distribution is integrated over time to obtain the intensity values $k_{\mathrm{sc}}(z, \rho)$, and added to the distribution of the unscattered photons $k_{\mathrm{usc}}(z, \rho)$ for the total distribution $k(z, \boldsymbol{\rho})($ Eq. 4$)$, called the BSF. Note that the equations also include absorption effects that were omitted from the figure for simplicity.

is adjacent to the slice surface, above the point of maximal intensity (Fig. 2c). Oblique, semi-coronal brain slices were obtained from two female mice (5 months old, C57/ BL6 strain) from the same litter. The slice thicknesses used were 150, 200, 300, 400, 500, and $600 \mu \mathrm{m}$. Four measurements per slice were taken in neocortical areas, with two to three slices of each thickness obtained per animal ( $n=16-20$ for each slice thickness). Because we found greater scattering near the white matter at the deep layers of the cortex, the measurements were performed at central locations. The slices and the fiber were submerged in artificial CSF throughout the measurements, and were oxygenated until shortly before the measurement. In addition, radial intensity profile was obtained for the 300- and 600- $\mu \mathrm{m}$-thick slices, by moving the fiber tip laterally in $50 \mu \mathrm{m}$ intervals to $1 \mathrm{~mm}$. The measured results were normalized to the measured light intensity without the slice (at the same height above the aperture) divided 
a

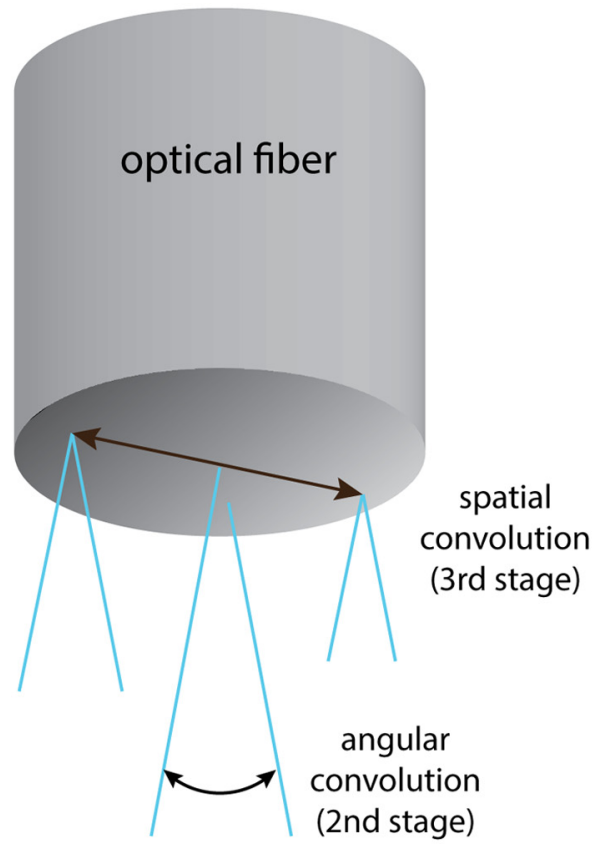

b

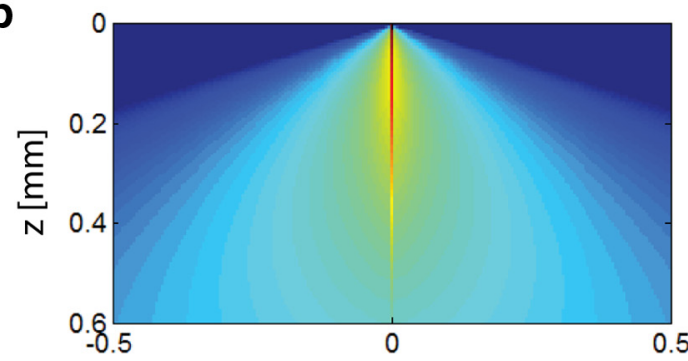

1 st

stage
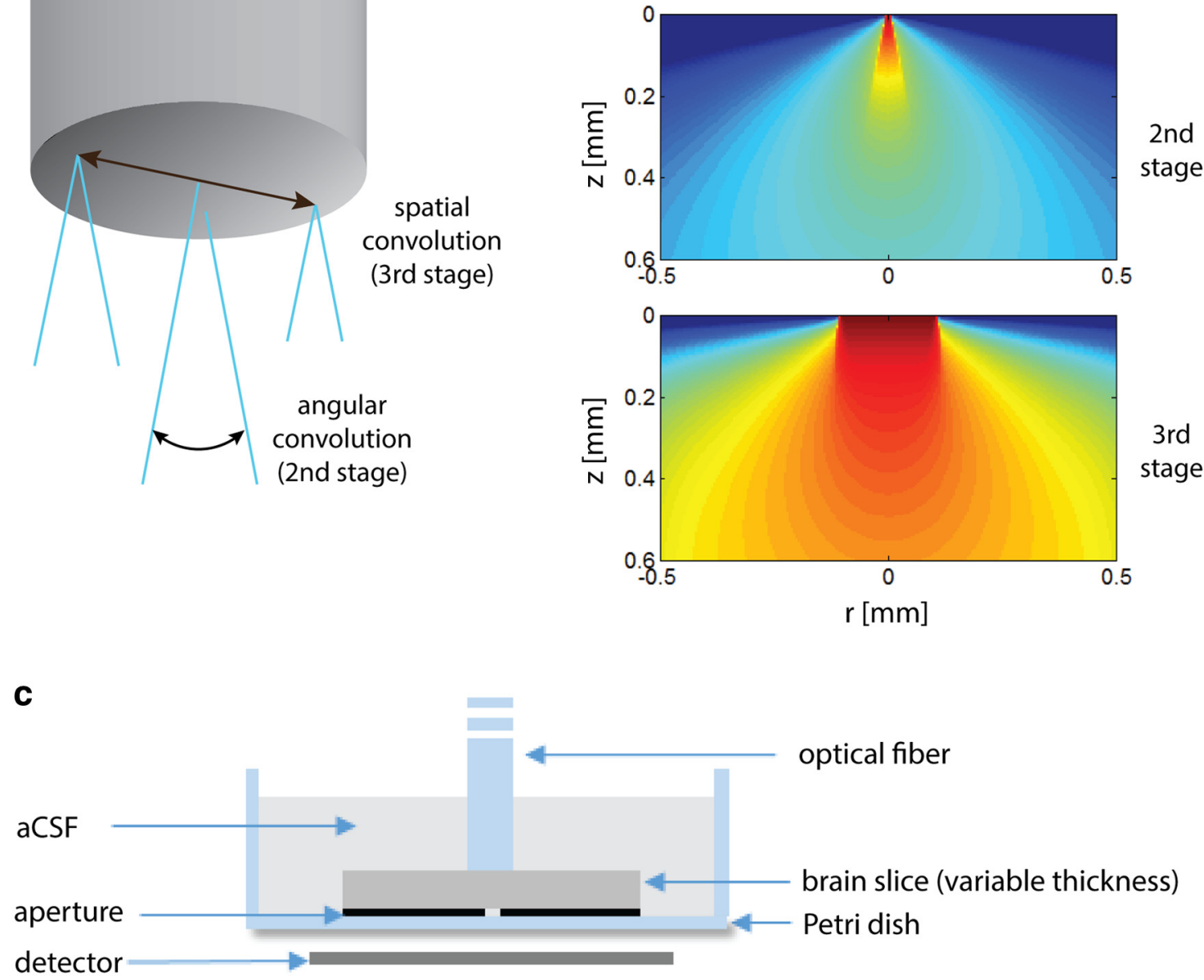

Figure 2. a, Simulation procedure: the 1st stage is the calculation of a 3D pencil-beam response, the 2 nd stage is the angular convolution of the pencil beam response, and the 3rd stage is a spatial convolution with the fiber tip area. $\boldsymbol{b}$, Simulation outcomes of the various simulation steps. For better visualization, all of the figures are in log scale. $\boldsymbol{c}$, Illustration of the experimental setup cross-section.

by the theoretical predicted attenuation due to geometric beam spreading.

\section{Results}

We first examined the accuracy of BSF method by comparing it to $\mathrm{MC}$ simulation results using two sets of previously published brain tissue optical parameters: scattering coefficient $\mu_{s}=168.6 \mathrm{~cm}^{-1}$ from Al-Juboori et al. (2013) measured in the mouse's pedunculopontine nucleus using $453 \mathrm{~nm}$ blue light and $\mu_{\mathrm{s}}=120 \mathrm{~cm}^{-1}$ from Yaroslavsky et al. (2002) measured in human brain graymatter tissue using $480 \mathrm{~nm}$ blue light. Figure $3 a$ shows contour maps of the calculated light transmission using the $\mathrm{MC}$ method, and Figure $3 b$ shows the same using the
BSF method. The fiber diameter used for the models in this figure is $100 \mu \mathrm{m}$ and the NA is 0.22 (as reported by Al-Juboori et al., 2013). Due to the lack of better data, we used the absorption coefficient and anisotropy factor of native human gray matter from Yaroslavsky et al. (2002): $\mu_{a}=0.6 \mathrm{~cm}^{-1}$ and $g=0.88$ in both cases. These results illustrate that the analytical BSF method generally follows the $\mathrm{MC}$ predictions quite closely for a parameter regime, which is relevant for optogenetics.

Next, we compared the models' predictions to two published experimental accounts on transmission along the $z$-axis, that is, the fiber's central axis (Fig. 3; Aravanis et al., 2007; Al-Juboori et al., 2013). First, we compared 
a

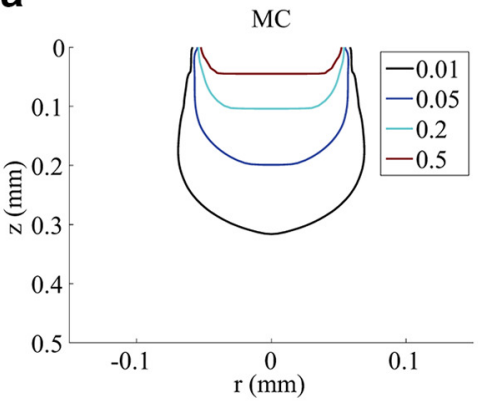

b

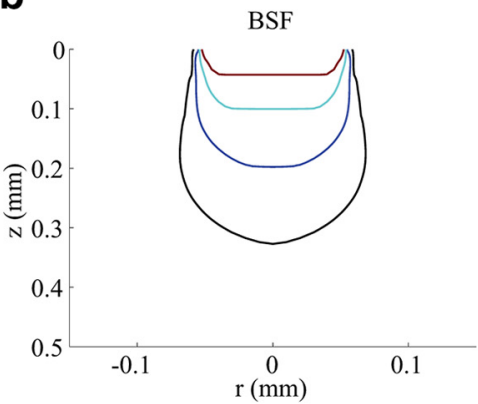

C

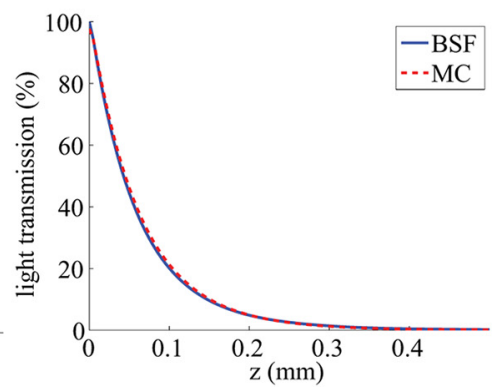

Top panels: scattering coefficient $168.6 \mathrm{~cm}^{-1}$, Al-Juboori et al. $(\lambda=453 \mathrm{~nm})$
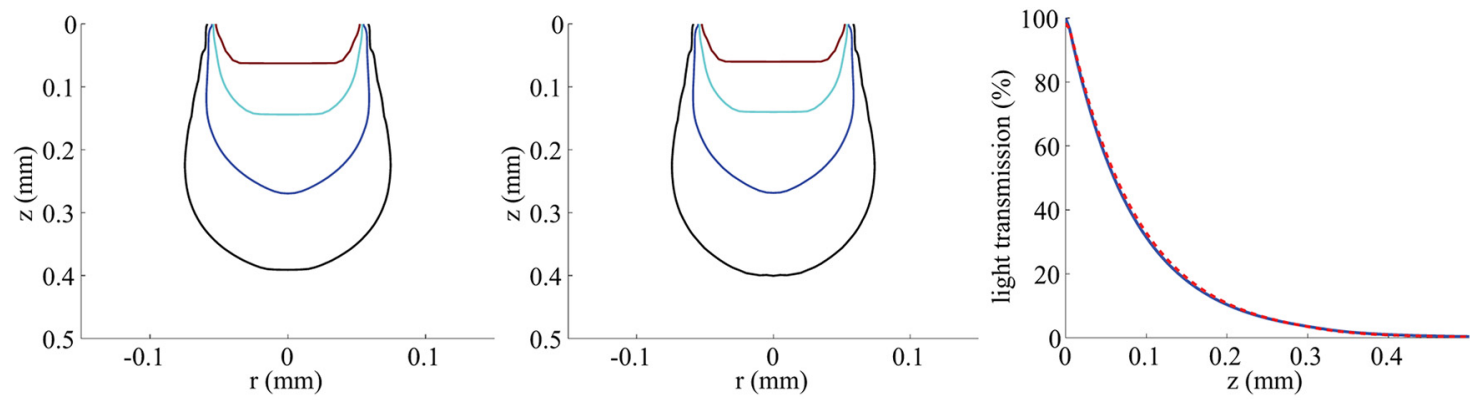

Bottom panels: scattering coefficient $120 \mathrm{~cm}^{-1}$, Yaroslavsky et al. $(\lambda=480 \mathrm{~nm})$

Figure. 3. Comparisons of MC and BSF methods for two different tissue-scattering parameter settings: $\mu_{s}=168.6 \mathrm{~cm}^{-1}$ (top; from Al-Juboori et al. 2013) and $\mu_{\mathrm{s}}=120 \mathrm{~cm}^{-1}$ (bottom; from Yaroslavsky et al. 2002). a, Contour maps of the light distribution in the tissue, created using the MC method. $\boldsymbol{b}$, Contours created using the BSF method. The iso-intensity lines are at $50,20,5$, and $1 \%$ of maximum. $\boldsymbol{c}$, Light transmission curves along the $z$-axis.

the models' results of Figure $3 c$ to the published decay curves by Al-Juboori et al., (2013; Fig. 4b). As discussed above, the results of the MC and the BSF methods are in excellent agreement [root mean square ( $\mathrm{rms}$ ) transmission error of $0.52 \%$ over the range $0-1 \mathrm{~mm}$, and we find that both provide a very good fit to the experimental measurements (rms error of $0.97 \%$ over the range $0-0.3 \mathrm{~mm}$ ). In contrast, the model-based simulations are in poor agree- ment with the experimental measurements of Aravanis et al. (2007). The comparison used the experimental setup geometry of Aravanis et al. (2007), where the fiber tip remained in a fixed height while the tissue samples were being replaced, and no aperture was used. It can be shown that the light attenuation curve obtained this way is equivalent to depicting the integrated light intensities over the entire layers. A version of the BSF model was adapted
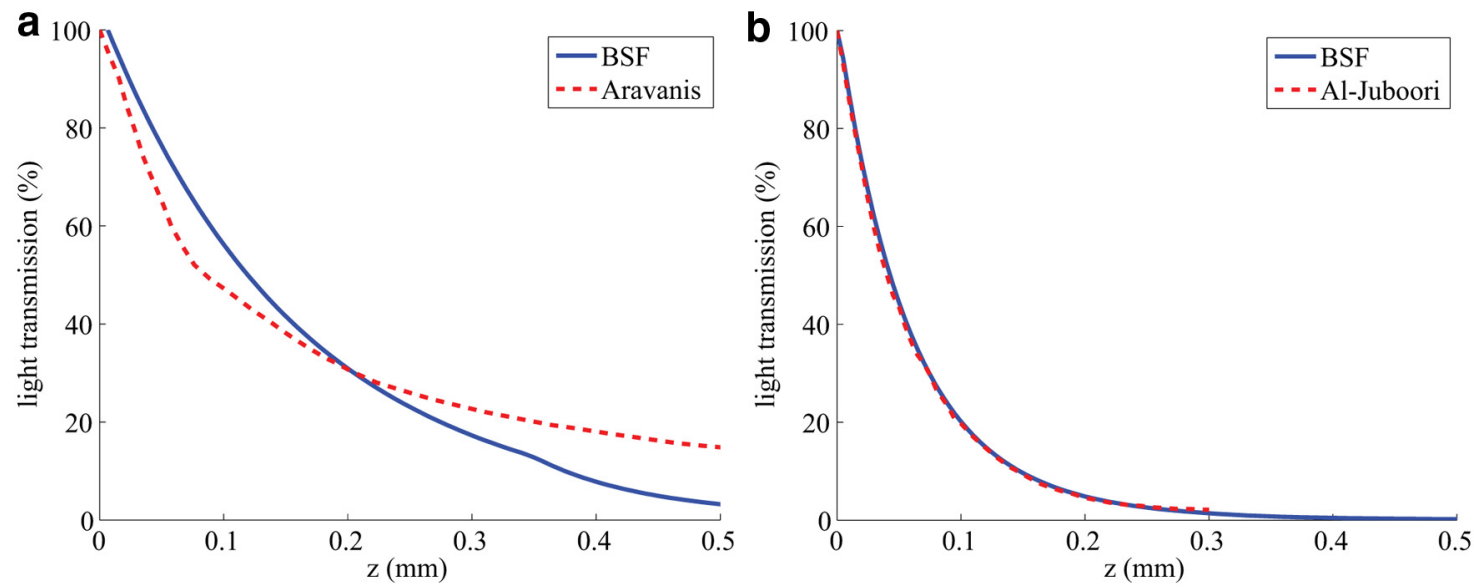

Figure. 4. Transmission of light along the $z$-axis, comparing published experimental results (red dotted curves) with BSF method (blue curves). $\boldsymbol{a}$, Best-fit curves to measured data from Aravanis et al. (2007). b. Experimental data from Al-Juboori et al. (2013) measured from the peduncopontine tegmental nucleus; the optical parameters used for the BSF curve are explained in the text. 
a

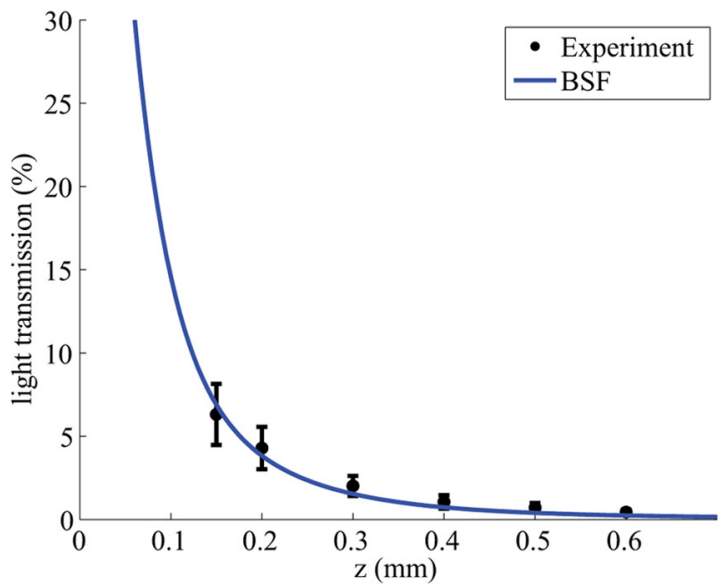

C

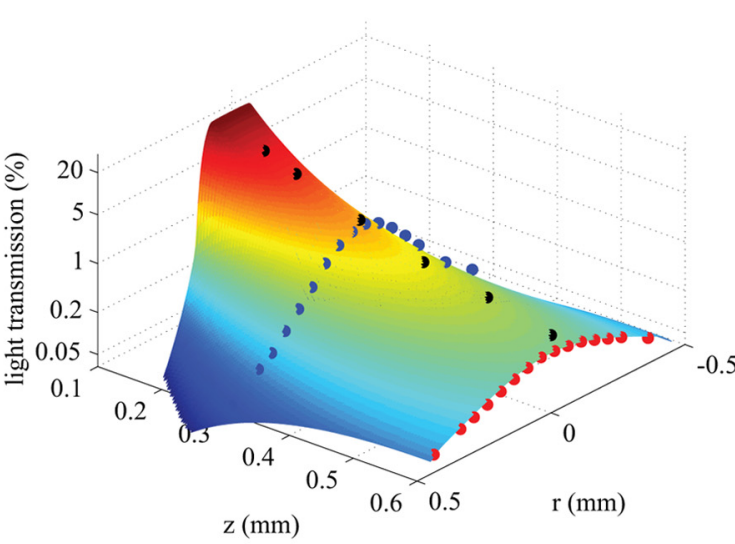

b

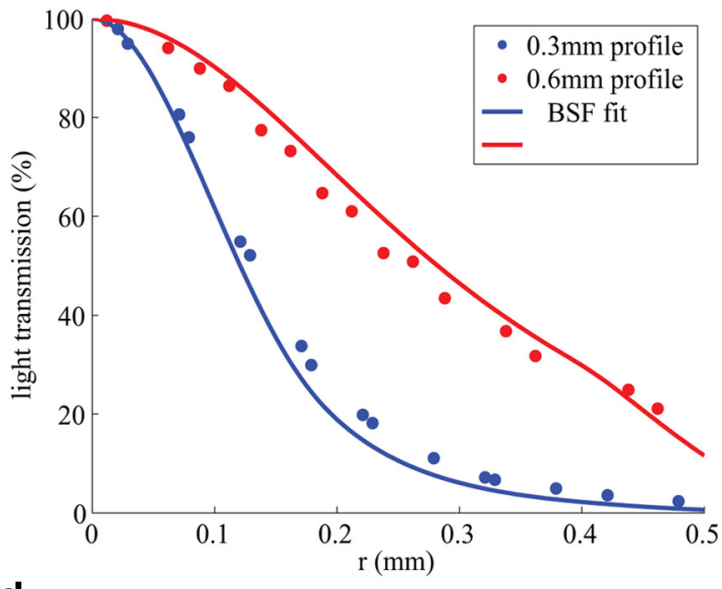

d

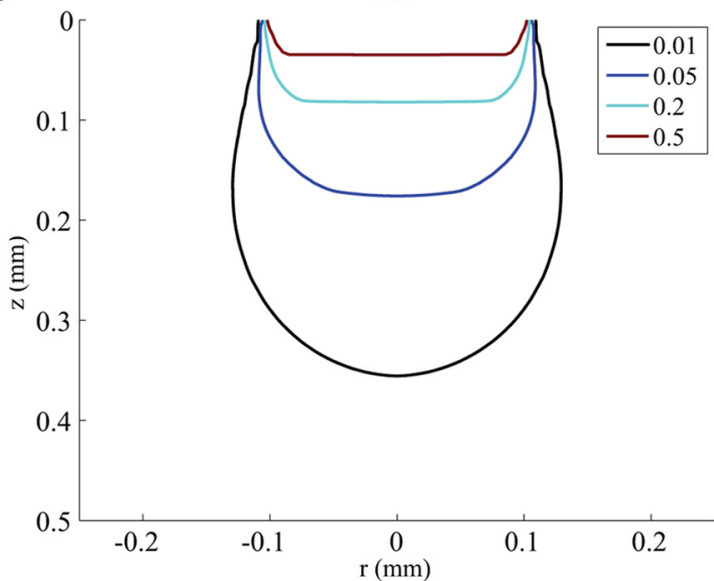

Figure. 5. Experimental results: light transmission in a mouse cortex along the $z$-axis (a) and the radial axis $(\mathbf{b})$ at $300 \mu \mathrm{m}(\mathrm{blue})$ and $600 \mu \mathrm{m}$ (red). The solid lines are the best fit of the BSF model. c, Surface plot of the simulated light distribution in the slice, obtained with BSF model using the experimentally estimated parameters $\left(\mu_{\mathrm{s}}=211 \mathrm{~cm}^{-1}, g=0.86\right)$, with overlaid experimental measurements (colors matched to $\boldsymbol{a}$ and $\boldsymbol{b}$ ). Light transmission is in log scale. $\boldsymbol{d}$, Contour map of the simulated light distribution.

to implement this integration, and best-fit curves to the data from Aravanis et al. (2007) used this modification. The obtained optical parameters were as follows: scattering coefficient $60.7 \mathrm{~cm}^{-1}$, absorption coefficient 0.62 $\mathrm{cm}^{-1}$, and anisotropy factor $g=0.89$.

Finally, we compared model-based predictions to the new set of measurements performed in adult mouse cortical slices (Fig. 5). To estimate the tissue's scattering parameters, we searched for a BSF model that simultaneously provided the best fit to both the axial and lateral profiles (obtained parameters: scattering coefficient 211 $\mathrm{cm}^{-1}$ and anisotropy factor $g=0.86$ ). The results again demonstrate an excellent agreement between the respective simulation methods, and between them and the experimental curves. Overall, these results exhibit a strong attenuation of the light intensity along the $z$-axis due to multiple scattering: the intensity is reduced to $50 \%$ at a depth $<40 \mu \mathrm{m}$, and to just a few percentages at depths exceeding $200 \mu \mathrm{m}$ (mean free path is $47 \mu \mathrm{m}$ ). This finding highlights how the gray matter's high density leads to a large number of scattering events over a relatively very short distance.

\section{Discussion}

In this study, we sought to develop and experimentally validate solutions for realistic modeling of optogenetic light delivery using an optical fiber embedded in brain tissue. MC simulations, a generally accepted numerical method for simulating light propagation in biological tissues (Zhu and Liu, 2013), have already been extensively applied to this problem, however, generally using inappropriate tissue parameters (Table 1) and without capturing the input light source's spatial and angular properties; these issues are particularly important in light of the general lack of experimental validation of simulation results. Additionally, we adapted and extended a powerful analytical method for estimating scattered light distributions, the BSF, and carefully compared it with the MC results and to experimental measurements. Importantly, the results of the analytical solutions were found to be highly consistent with the MC simulation results, with the published attenuation profile measurements by Al-Juboori et al. (2013) in mouse subcortical regions (Fig. 4b), and with a new set of cortical attenuation measurements (Fig. 
Table 1. Brain tissue optical parameters

\begin{tabular}{|c|c|c|c|c|}
\hline Source & Brain sample & Wavelength, nm & Scattering coefficient, $\mathrm{cm}^{-1}$ & Anisotropy factor \\
\hline Our experiment & Adult mouse cortex, (5 months old) & 473 & 211 & 0.86 \\
\hline Al-Juboori et al. (2013) & Mouse subcortical, (6-8 weeks old) & 453 & 168.6 & - \\
\hline Yaroslavsky et al. (2002) & Human gray matter & 480 & 120 & 0.88 \\
\hline
\end{tabular}

5). The model-based iso-intensity contours in cortical slices (Fig. $5 d$ ) are also in rough agreement with the measured results found in Yizhar et al. (2011, their Fig. $3 \mathrm{E})$, which is itself in stark disagreement with the attenuation graph of Aravanis et al. (2007; reproduced by Yizhar et al. 2011, their Fig. 3B). Our method for measuring and estimating the parameters uses a geometry and fits to localized measurements that are directly relevant to optogenetics and does not rely on the multiple assumptions behind diffuse reflectance estimates (Azimipour et al. 2014).

The very good inter-model agreement found is probably as good as one can obtain, given that there are several reasons to expect small discrepancies between the two methods. First, the BSF formulation uses several approximations and assumptions. For example, the temporal probability density is modeled as a Gamma distribution, although more recent work (Funk and Pfeilsticker, 1999; Theer and Denk, 2006) suggests the lognormal distribution may be a more accurate model. Moreover, the methods have some inherent numerical errors, especially as a result of integration, interpolations, and the limited volume (spatial, temporal, and directional) allocated for the Green function. Likewise, differences from the empirical measurements can be attributed to tissue inhomogeneity, approximations in the experimental setup (e.g., neglecting the polystyrene and air layers between the slice and the detector, errors in slice thickness, etc), and errors in the estimated optical parameters used. Finally, although the methods were applied here to a uniform light source, it can be easily accommodated to other beam profiles (e.g., Gaussian beam, multiple fibers, etc). It is, however, important to note that like almost all current methods that describe the propagation of light in a turbid media, both

Table 2. The illuminated tissue volumes for various commercial optical fibers

\begin{tabular}{|c|c|c|c|c|c|}
\hline \multicolumn{2}{|c|}{ Fiber properties } & \multicolumn{4}{|c|}{$\begin{array}{l}\text { Volume illuminated } \\
\left(10^{-3} \times \mathrm{mm}^{3}\right), \%\end{array}$} \\
\hline NA & Diameter, $\mu \mathrm{m}$ & $>50$ & $>20$ & $>5$ & $>1$ \\
\hline 0.1 & 25 & 0.02 & 0.04 & 0.09 & 0.20 \\
\hline 0.22 & 50 & 0.06 & 0.14 & 0.34 & 0.79 \\
\hline 0.22 & 105 & 0.29 & 0.68 & 1.48 & 3.28 \\
\hline 0.22 & 200 & 1.07 & 2.56 & 5.53 & 13.7 \\
\hline 0.22 & 365 & 3.62 & 8.71 & 19.5 & 61.7 \\
\hline 0.37 & 200 & 1.05 & 2.52 & 5.52 & 14.3 \\
\hline 0.39 & 200 & 1.05 & 2.51 & 5.50 & 14.4 \\
\hline 0.39 & 300 & 2.39 & 5.75 & 12.7 & 38.1 \\
\hline 0.39 & 400 & 4.30 & 10.4 & 23.3 & 79.4 \\
\hline 0.48 & 400 & 4.76 & 10.3 & 23.1 & 79.0 \\
\hline
\end{tabular}

Threshold is in percentage of maximal illumination. methods do not take the wave properties of the light into account; thus, when using a coherent source, the interference of photons with different phases (due to different optical path lengths) will form a speckle pattern that can also reduce the total illumination level at each position.

Using the new BSF method, the scattering coefficient and anisotropy factor were estimated in measurements performed in mouse cortical slices. This estimation procedure was based on simultaneously optimizing the mean squared error (MSE) fit to both the axial and two lateral curves (Fig. 5) using only two scattering parameters (or "degrees of freedom"). This iterative fit procedure was facilitated by the tremendous speed gain of the BSF method relative the $\mathrm{MC}$ numerical simulations $(\sim 10 \mathrm{sec}$ per BSF computation versus $\sim 10 \mathrm{hr}$ per MC to achieve a comparable accuracy on a modern desktop PC).

Table 1 compares our results to estimates of these parameters based on the published data by Aravanis et al. (2007), as well as related parameter estimates obtained in the adult mouse pedunculopontine tegmental nucleus (AlJuboori et al., 2013), in adult rat cortex (Fig. 5; Azimipour et al., 2014) and in human gray matter (Yaroslavsky et al., 2002). This comparison illustrates the much stronger scattering in mouse cortex seen in our results than indicated by Aravanis et al. (2007) and is generally more consistent with the stronger scattering observed by $\mathrm{Al}-$ Juboori et al. (2013) and Azimipour et al. (2014). Noting the considerable difference in the optical parameters between human and mouse brains, and between brain areas, we advise to refrain from using them interchangeably. Indeed, these findings explain the observations in Bernstein et al. (2008), who report a ratio of $\sim 0.73$ in the diameters of the $1 \%$ and $10 \%$ contours of light attenuation between their measurements in mouse brain slices and $\mathrm{MC}$ simulations, which were based on human brain optical parameters. Note, however, that these estimates should be used with caution if scattering is expected to change. For example, measurements should be performed on tissue samples obtained from younger animals, as their scattering has been shown to be weaker (Oheim et al., 2001).

These results therefore put forward the BSF as a viable analytical approach toward a better understanding of light propagation and interactions in optogenetics and related fields, whereas use of the Kubelka-Munk model should be discouraged due to its theoretical inadequacy. As we have shown, the BSF can be used both for calculating the light distribution in brain tissue, and for estimating the optical parameters from measured attenuation curves. Because the BSF is an analytical method, it is considerably faster than a MC simulation, enabling its implemen- 
tation to curve fitting algorithms that obtain the optical parameters of the tissue and require multiple calculations of the attenuation curve with different parameters. A major limitation of light propagation models in brain tissue (including the ones portrayed here) is the assumption of homogeneity, whereas the cortical layers have different cytological properties, and necessarily also different optical parameters. These can be obtained in a similar manner, by performing the attenuation curve fitting in parts using the histologic layers' depths. A useful, immediate application of the BSF method for experiment design is to determine the tissue volume illuminated to various levels by different optical fibers (Table 2). It is noted that exact calculation of the light intensity inside the tissue is a prerequisite for determining the excitation from optogenetic stimulation, but does not completely describe the excitation from optogenetic light. To estimate the excitation properly, one has to know the cell morphology, the distribution of the light-sensitive channels and the baseline excitability from subthreshold oscillations. Moreover, in vivo optogenetic stimulation is also prone to absorption by blood (Azimipour et al., 2015), which is highly nonhomogenous and remains untreated in our model.

A BSF solver is available at: niel.net.technion.ac.il/ software.

\section{References}

Adamantidis AR, Zhang F, Aravanis AM, Deisseroth K, de Lecea L (2007) Neural substrates of awakening probed with optogenetic control of hypocretin neurons. Nature 450:420-424. CrossRef Medline

Al-Juboori S, Dondzillo A, Stubblefield EA, Felsen G, Lei TC, Klug A (2013) Light scattering properties vary across different regions of the adult mouse brain. PLoS One 8:e67626. CrossRef Medline

Aravanis AM, Wang LP, Zhang F, Meltzer LA, Mogri MZ, Schneider MB, Deisseroth K (2007) An optical neural interface: in vivo control of rodent motor cortex with integrated fiberoptic and optogenetic technology. J Neural Eng 4:S143-S156. CrossRef Medline

Azimipour M, Baumgartner R, Liu Y, Jacques SL, Eliceiri K, Pashaie $R$ (2014) Extraction of optical properties and prediction of light distribution in rat brain tissue. J Biomed Opt 19:075001. CrossRef Medline

Azimipour M, Atry F, Pashaie R (2015) Effect of blood vessels on light distribution in optogenetic stimulation of cortex. Opt Lett 40:21732176. Medline

Beck A, Teboulle M (2009) A fast iterative shrinkage-thresholding algorithm for linear inverse problems. SIAM J Img Sci 2:183-202. CrossRef

Bernstein JG, Han X, Henninger MA, Ko EY, Qian X, Franzesi GT, McConnell JP, Stern P, Desimone R, Boyden ES (2008) Prosthetic systems for therapeutic optical activation and silencing of genetically-targeted neurons. Proc SPIE Int Soc Opt Eng 6854: $68540 \mathrm{H}-68540 \mathrm{H}$.
Chow BY, Han X, Dobry AS, Qian X, Chuong AS, Li M, Henninger MA, Belfort GM, Lin Y, Monahan PE, Boyden ES (2010) Highperformance genetically targetable optical neural silencing by light-driven proton pumps. Nature 463:98-102. CrossRef Medline

Deisseroth K (2011) Optogenetics. Nature Methods 8:26-29. CrossRef Medline

Deisseroth K, Schnitzer M (2013) Engineering approaches to illuminating brain structure and dynamics. Neuron 80:568-577. CrossRef Medline

Fang Q (2010) Mesh-based Monte Carlo method using fast raytracing in plucker coordinates. Biomed Opt Express 1:165-175. CrossRef Medline

Foutz TJ, Arlow RL, Mclntyre CC (2012) Theoretical principles underlying optical stimulation of a channelrhodopsin-2 positive pyramidal neuron. J Neurophysiol 107:3235-3245. CrossRef Medline

Frigault MM, Lacoste J, Swift JL, Brown CM (2009) Live-cell microscopy: tips and tools. J Cell Sci 122:753-767. CrossRef Medline

Funk O, Pfeilsticker K (1999) Photon path length distributions for cloudy skies - oxygen A-band measurements and model calculations. Ann Geophys 21:615-626. CrossRef

Kahn I, Desai M, Knoblich U, Bernstein J, Henninger M, Graybiel AM, Boyden ES, Buckner RL, Moore Cl (2011) Characterization of the functional MRI response temporal linearity via optical control of neocortical pyramidal neurons. J Neurosci 31:15086-15091. CrossRef Medline

Kubelka P (1948) New contributions to the optics of intensely lightscattering materials. part I. J Opt Soc Am 38:448-457. Medline

McLean JW, Freeman JD, Walker RE (1998) Beam spread function with time dispersion. Appl Opt 37:4701-4711. Medline

Neuman M, Edström P (2010) Anisotropic reflectance from turbid media: I. Theory. J Opt Soc Am A Opt Image Sci Vis 27:10321039.

Oheim M, Beaurepaire E, Chaigneau E, Mertz J, Charpak S (2001) Two-photon microscopy in brain tissue: Parameters influencing the imaging depth. J Neurosci Methods 111:29-37. Medline

Okamoto K (2006) Fundamentals of optical waveguides. Burlington, MA: Elsevier.

Pashaie R, Anikeeva P, Jin Hyung Lee, Prakash R, Yizhar O, Prigge M, Chander D, Richner TJ, Williams J (2014) Optogenetic brain interfaces. IEEE Rev Biomed Eng 7:3-30. CrossRef Medline

Theer P, Denk W (2006) On the fundamental imaging-depth limit in two-photon microscopy. J Opt Soc Am A Opt Image Sci Vis 23:3139-3149. Medline

Vo-Dinh T (2003) Biomedical photonics handbook. Boca Raton, FL: CRC.

Yaroslavsky AN, Schulze PC, Yaroslavsky IV, Schober R, Ulrich F, Schwarzmaier HJ (2002) Optical properties of selected native and coagulated human brain tissues in vitro in the visible and near infrared spectral range. Phys Med Biol 47:2059-2073. Medline

Yizhar O, Fenno LE, Davidson TJ, Mogri M, Deisseroth K (2011) Optogenetics in neural systems. Neuron 71:9-34. CrossRef Medline

Zhang F, Vierock J, Yizhar O, Fenno LE, Tsunoda S, Kianianmomeni A, Prigge M, Berndt A, Cushman J, Polle J, Magnuson J, Hegemann P, Deisseroth K (2011) The microbial opsin family of optogenetic tools. Cell 147:1446-1457. CrossRef Medline

Zhu C, Liu Q (2013) Review of Monte Carlo modeling of light transport in tissues. J Biomed Opt 18:50902. CrossRef Medline 